\title{
The existence problem for dynamics of dissipative systems in quantum probability
}

\author{
Palle E. T. Jorgensen* \\ Department of Mathematics, The University of Iowa, \\ 14 MacLean Hall, Iowa City, IA 52242-1419 U.S.A.
}

(Dated: November 20, 2018)

\begin{abstract}
Motivated by existence problems for dissipative systems arising naturally in lattice models from quantum statistical mechanics, we consider the following $C^{*}$-algebraic setting: A given hermitian dissipative mapping $\delta$ is densely defined in a unital $C^{*}$-algebra $\mathfrak{A}$. The identity element in $\mathfrak{A}$ is also in the domain of $\delta$. Completely dissipative maps $\delta$ are defined by the requirement that the induced maps, $\left(a_{i j}\right) \rightarrow\left(\delta\left(a_{i j}\right)\right)$, are dissipative on the $n$ by $n$ complex matrices over $\mathfrak{A}$ for all $n$. We establish the existence of different types of maximal extensions of completely dissipative maps. If the enveloping von Neumann algebra of $\mathfrak{A}$ is injective, we show the existence of an extension of $\delta$ which is the infinitesimal generator of a quantum dynamical semigroup of completely positive maps in the von Neumann algebra. If $\delta$ is a given well-behaved $*$-derivation, then we show that each of the maps $\pm \delta$ is completely dissipative.
\end{abstract}

PACS numbers: 02., 02.10.Hh, 02.30.Tb, 03.65.-w, 05.30.-d

Keywords: spin systems, dynamics, evolution semigroup, dissipative, $C^{*}$-algebra, noncommutative probability

*Electronic address: jorgen@math.uiowa.edu URL: http://www.math.uiowa.edu/〜jorgen/ Supported in part by the National Science Foundation under grants DMS-9987777 and DMS-0139473 (FRG). 


\section{INTRODUCTION}

Recent applications of the operator-theoretic approach to dissipative quantum systems include [22] and 37]. For a more systematic approach, see [36]. Suppose we are given a one-parameter group of automorphisms $\alpha_{t}: a \mapsto e^{i t H} a e^{-i t H}$ which acts on some set of observables $a$, specified as a dense "local" subalgebra of a completed $C^{*}$-algebra. If we then differentiate at $t=0$, we get the derivation $\delta: a \mapsto i[H, a]=i(H a-a H)$ which takes the form of a formal commutator. The issue is complicated by the fact that the Hamiltonian $H$ is typically an unbounded operator in statistical models, say infinite lattice spin systems. In applications, it is $H$ that is given, and the process must be run in reverse. By analogy to boundary value problems from partial differential equations, we then expect to encounter an existence problem for reconstructing the dynamics of the system from knowing only a formula for $H$.

We adopt the $C^{*}-W^{*}$-formalism for the dynamics of infinite quantum systems $\llbracket 10,14$, 18, 20, 21, 24, 31, 33]. For the special case of quantum spin systems it is believed that the dynamics in the time reversible case is given by an unbounded derivation of a suitable algebra $\mathfrak{A}$ of observables [31]. Depending on the range of the interaction, and the number of dimensions of the spin lattice, it is possible to exponentiate the infinitesimal derivation to a one-parameter group of automorphisms $\alpha_{t}(-\infty<t<\infty)$ of $\mathfrak{A}$, or of the enveloping $W^{*}$-algebra $\mathfrak{A}^{\prime \prime}$ (see [35]), or the $W^{*}$-algebra generated by a given invariant state [10, 17, 26, 29, 32,33$]$.

It is known that (open) irreversible systems may be obtained as restrictions of timereversible systems, and it follows 20] that the dynamics of the open system is given mathematically by a semigroup $\tau_{t}(0 \leq t<\infty)$ of completely positive mappings of the $C^{*}$-algebra $\mathfrak{A}$, or $W^{*}$-algebra $\mathfrak{A}^{\prime \prime}$. The corresponding infinitesimal generator is completely dissipative. Completely positive semigroups also play a role in quantum computing algorithms [27]. The philosophy is that noise in the quantum processes dictates the dissipative systems, as opposed to the conservative ones (which are governed by one-parameter groups of automorphisms).

But in high lattice-dimensions, or for long-range interaction, there are difficulties in exponentiating the infinitesimal generators. The determination of the time-evolutions $\alpha_{t}$ (resp., $\tau_{t}$ ) seems to require "extra boundary conditions" [7, 10, 26, 32, 33]. It is therefore a meaningful foundational question, for a given completely dissipative infinitesimal transformation 
$\delta$ in a $C^{*}$-algebra $\mathfrak{A}$, to ask if it is always possible to extend $\delta$ to a transformation $\tilde{\delta}$ which is the infinitesimal generator for a quantum dynamical semigroup. Under the assumption that $\delta$ is hermitian, and that the $W^{*}$-algebra $\mathfrak{A}^{\prime \prime}$ is injective, we establish the existence of a generator extension $\tilde{\delta}$. Our extension is thus an algebraic parallel to Friederichs's extension for semibounded operators in Hilbert space, or an analogue of Phillips's [30] maximal dissipative extension of the general dissipative operator in Hilbert space.

In earlier articles [7, 10, 28, 31] the uniqueness problem was considered for the generator extension, $\delta \subset \tilde{\delta}$. But, just as is the case for operators in Hilbert space (Friedrichs, Phillips), the extension is generally not unique, reflecting the possibility of different "boundary conditions" at infinity.

We refer the reader to the books [14], 18] and [33] for details on the mathematical foundations of algebraic quantum theory.

The issues centering around the existence problem for the dynamical one-parameter groups, or semigroups, of quantum statistical mechanics are perhaps best known in the setup of quantum spin systems, as they are treated in [9], 25] and 34].

Example I.1. The mathematical framework is rather general such as to allow a wide variety of applications, including recent ones to nonequilibrium statistical mechanics [34]. A countably infinite set $L$ (say a lattice; it may be $\mathbb{Z}^{\nu}$ where $\nu$ is the lattice rank, or dimension) is specified at the outset. Points $s \in L$ are sites at which quantum spins are located. For each $s \in L$, let $\mathcal{H}_{s}$ be a finite-dimensional complex Hilbert space, i.e., the spin vectors at site $s$; and for a finite subset $\Lambda \subset L$, set

$$
\mathcal{H}_{\Lambda}:=\bigotimes_{s \in \Lambda} \mathcal{H}_{s}
$$

Then let $\mathfrak{A}_{\Lambda}$ be the $*$-algebra of all (bounded) operators on $\mathcal{H}_{\Lambda}$. With the natural embedding

$$
\mathfrak{A}_{\Lambda_{1}} \subset \mathfrak{A}_{\Lambda_{2}} \quad \text { for } \Lambda_{1} \subset \Lambda_{2}
$$

given by

$$
\mathfrak{A}_{\Lambda_{1}} \longmapsto \mathfrak{A}_{\Lambda_{1}} \otimes 1_{\Lambda_{2} \backslash \Lambda_{1}} \subset \mathfrak{A}_{\Lambda_{2}}
$$

we get the usual inductive limit $C^{*}$-algebra $\lim _{\Lambda} \mathfrak{A}_{\Lambda}=$ : $\mathfrak{A}$. A function $\Lambda \mapsto \Phi(\Lambda)=\Phi(\Lambda)^{*} \in$ $\mathfrak{A}_{\Lambda}$ defined on the finite subsets $\Lambda$ of $L$ is called an interaction, and

$$
H_{\Phi}(\Lambda)=\sum_{X \subset \Lambda} \Phi(X)
$$


is the associated local Hamiltonian, where in (I.1), the summation is over all finite subsets $X$ of $\Lambda$. Since $\mathfrak{A}_{\Lambda_{1}}$ and $\mathfrak{A}_{\Lambda_{2}}$ commute when $\Lambda_{1} \cap \Lambda_{2}=\varnothing$, it follows that

$$
\delta(a)=\lim _{\Lambda}[H(\Lambda), a]
$$

is well defined for all local observables $a$ in the dense $*$-subalgebra

$$
\mathfrak{A}_{0}=\bigcup_{\Lambda \text { fin }} \mathfrak{A}_{\Lambda} \quad \text { in } \mathfrak{A}
$$

where $[\cdot, \cdot]$ in (I.2) denotes the usual commutator $[b, a]:=b a-a b$. Ruelle proved that, if $\Phi$ is translationally invariant, and if, for some $\lambda>0$,

$$
\sum_{n=0}^{\infty} e^{n \lambda} \sup _{s \in L} \sum_{\substack{s \in X \text { fin } \\ \operatorname{card} X=n+1}}\|\Phi(X)\|<\infty,
$$

then the $*$-derivation $\delta$ defined in (I.2) is the infinitesimal generator of a one-parameter subgroup of $*$-automorphisms $\left\{\alpha_{t}\right\}_{t \in \mathbb{R}} \subset$ Aut $(\mathfrak{A})$, which then satisfies

$$
\alpha_{t}(a)=\lim _{\Lambda \nearrow L} e^{i t H(\Lambda)} a e^{-i t H(\Lambda)}
$$

for all $a \in \mathfrak{A}$ and $t \in \mathbb{R}$, i.e., it is approximately inner. This means that, if $a \in \mathfrak{A}_{0}$, then

$$
\lim _{\substack{t \rightarrow 0 \\ t \neq 0}} t^{-1}\left(\alpha_{t}(a)-a\right)=\delta(a) .
$$

Moreover, $\delta$ is, when extended from $\mathfrak{A}_{0}$, a closed $*$-derivation, in the sense that the graph of $\delta$ is closed in $\mathfrak{A} \times \mathfrak{A}$. But if $\Phi$ is not translationally invariant, or if (1.3) is not known to hold, then no such conclusion is within reach, and the issue of extensions of $\delta$ arises. We

then ask if some extension $\tilde{\delta}$ of $\delta$ to a generator of a one-parameter group of automorphisms, or a semigroup of dissipations (see details below), exists.

\section{DEFINITIONS AND TERMINOLOGY}

Let $X$ and $Y$ be Banach spaces. Then the space of bounded linear operators from $X$ to $Y$ is denoted $L(X, Y)$. The conjugate (i.e., dual) Banach space to $X$ is $L(X, \mathbb{C})$, and is denoted $X^{\prime}$. If $\mathcal{H}$ is a Hilbert space, the $C^{*}$-algebra of all bounded operators on $\mathcal{H}$ is denoted $B(\mathcal{H})$. Let $\mathcal{L}$ be a linear subspace of $B(\mathcal{H})$ which is self-adjoint and contains the identity operator $I$. With the order inherited from $B(\mathcal{H})$, the subspace $\mathcal{L}$ gets the structure of an 
operator system, in the terminology of Effros [11]. The full matrix algebra $M_{n}$ of all complex $n$-by- $n$ matrices is also an operator system, and so is $\mathcal{L}_{n}=\mathcal{L} \otimes M_{n}$. The elements in $\mathcal{L}_{n}$ may be realized as $n$-by- $n$ matrices with entries from $\mathcal{L},\left(a_{i j}\right)_{i, j=1}^{n}, a_{i j} \in \mathcal{L}$. If $\mathcal{L}$ and $\mathcal{R}$ are operator systems and $\varphi: \mathcal{L} \rightarrow \mathcal{R}$ is a linear mapping, then the induced map $\left(a_{i j}\right) \rightarrow\left(\varphi\left(a_{i j}\right)\right)$ of $\mathcal{L}_{n}$ into $\mathcal{R}_{n}$ is denoted $\varphi_{n}$. It is, in fact, $\varphi \otimes \mathrm{id}_{n}$. We say 2] that $\varphi$ is completely positive (resp., completely contractive) if $\varphi_{n}$ is positive (resp., contractive) for all $n$. We say that $\mathcal{R}$ is injective if for every pair of operator systems, $\mathcal{L} \subset \mathcal{L}_{1}$, and every completely positive map $\varphi: \mathcal{L} \rightarrow \mathcal{R}$, there is a completely positive extension $\psi: \mathcal{L}_{1} \rightarrow \mathcal{R}$. That is, $\psi(x)=\varphi(x)$ for all $x \in \mathcal{L}$. If $\mathcal{R}$ is a von Neumann algebra in a Hilbert space $\mathcal{H}$, it is known [13, 38] that $\mathcal{R}$ is injective iff there is a norm-one projection of $B(\mathcal{H})$ onto $\mathcal{R}$.

If $\mathfrak{A}$ is a $C^{*}$-algebra, it is known [12] that $\mathfrak{A}$ is nuclear iff the double conjugate (dual) $\mathfrak{A}^{\prime \prime}$ is injective as a $W^{*}$-algebra. Connes showed [13] that a factor $\mathcal{R}$ on a separable Hilbert space is injective iff it is matricial.

\section{DISSIPATIVE TRANSFORMATIONS}

An operator $\delta$ in a Banach space $X$ is said to be dissipative [29] if one of the following three equivalent conditions is satisfied:

(i) For all $x$ in the domain $\mathcal{D}(\delta)$ of $\delta$, there is an element $f \in X^{\prime}$, depending on $x$, such that $\|f\|=1, f(x)=\|x\|$, and $\operatorname{Re} f(\delta(x)) \leq 0$.

(ii) For all $x$ in $\mathcal{D}(\delta)$, and all $f \in X^{\prime}$ satisfying $\|f\|=1$, and $f(x)=\|x\|$, the inequality $\operatorname{Re} f(\delta(x)) \leq 0$ is valid.

(iii) For all $x$ in $\mathcal{D}(\delta)$, and all $\alpha \in \mathbb{R}_{+}$, the inequality $\|x-\alpha \delta(x)\| \geq\|x\|$ holds.

The proof of the equivalence can be found, for example, in [7], but the equivalence can also be shown to be a consequence of the approximation idea in Section $I X$ and Proposition $\mathrm{X} .2$ in the present paper.

If $X$ is an operator system, we say that $\delta$ is completely dissipative if the induced mapping $\delta_{n}$ in $X_{n}$ is dissipative for all $n=1,2, \ldots$ Recall that $X_{n}=X \otimes M_{n}$, and $\delta_{n}:\left(x_{i j}\right) \rightarrow\left(\delta\left(x_{i j}\right)\right)$, with domain $\mathcal{D}\left(\delta_{n}\right)=\left\{\left(x_{i j}\right) \in X_{n}: x_{i j} \in \mathcal{D}(\delta)\right\}$.

Finally we say that the transformation $\delta$ is hermitian if the domain $\mathcal{D}(\delta)$, in the operator system $X$, is invariant under the $*$-involution of $X$, and if $\delta\left(x^{*}\right)=\delta(x)^{*}$ for all $x \in \mathcal{D}(\delta)$. 
If $\delta: X \rightarrow Y$ is merely a linear transformation between Banach spaces $X$ and $Y$, with domain $\mathcal{D}(\delta)$ dense in $X$, then the transposed (or conjugate) transformation $\delta^{\prime}$ is well defined as a linear transformation $\delta^{\prime}: Y^{\prime} \rightarrow X^{\prime}$ with domain $\mathcal{D}\left(\delta^{\prime}\right)=\left\{f \in Y^{\prime}: \exists g \in X^{\prime}\right.$ s.t. $f(\delta(x))=g(x)$ for all $x \in \mathcal{D}(\delta)\}$. For $f \in \mathcal{D}\left(\delta^{\prime}\right), \delta^{\prime}(f)=g$. The domain $\mathcal{D}\left(\delta^{\prime}\right)$ is weak ${ }^{*}$ dense in $Y^{\prime}$ iff $\delta$ is closable. It is known [29] that dissipative operators are closable.

\section{COMPLETELY POSITIVE SEMIGROUPS (QUANTUM DYNAMICAL SEMIGROUPS)}

Let $M$ be a $W^{*}$-algebra with predual $M_{*}$. Let $\tau_{t}$ be a family of completely positive mappings of $M$ into itself, indexed by the time parameter $t \in[0, \infty)$. Assume that $\tau_{0}$ is the identity transformation in $M$, and that $\tau_{t}(\mathbb{1})=\mathbb{1}$ for all $t \in[0, \infty)$, where $\mathbb{1}$ denotes the unit element of the $W^{*}$-algebra $M$ in question. We assume further that the semigroup law holds, $\tau_{t_{1}+t_{2}}=\tau_{t_{1}} \circ \tau_{t_{2}}$ for $t_{1}, t_{2} \in[0, \infty)$, and finally that each $\tau_{t}$ is a normal mapping in $M$. Recall that normality is equivalent to the requirement that the conjugate semigroup $\tau_{t}^{\prime}$ [16] of $M^{\prime}$ leaves invariant the subspace $M_{*}$. Finally we require continuity of each scalar function, $t \rightarrow \varphi\left(\tau_{t}(a)\right)$, for all $\varphi \in M_{*}$ and $a \in M$. A semigroup which satisfies all the requirements above is called a completely positive semigroup. Because of the relevance to quantum dynamics, we shall also call it a quantum dynamical semigroup [21].

The infinitesimal generator of a given completely positive semigroup $\left(\tau_{t}, M\right)$ is a, generally unbounded, transformation, denoted by $\zeta$, in $M$. The domain of the generator $\zeta$ is given by

$$
\mathcal{D}(\zeta)=\left\{a \in M: \exists b \in M \text { s.t. for all } t, \tau_{t}(a)-a=\int_{0}^{t} \tau_{s}(b) d s\right\}
$$

By definition $\zeta(a)=b$. It is easy to see [16] that $\zeta(a)=\left.\frac{d}{d t} \tau_{t}(a)\right|_{t=0}$, where the derivative is taken in the $\sigma\left(M, M^{*}\right)$-topology. Finally note that infinitesimal generators are completely dissipative.

Example IV.1. It is known that the generator $\delta$ of a completely positive semigroup $\left\{\tau_{t}\right\}_{t \in \mathbb{R}_{+}}$ on a $C^{*}$-algebra $\mathfrak{A}$ is completely dissipative on a dense subspace $\mathcal{D}$ in $\mathfrak{A}$; see [4]. The following is a "canonical" example of this: it is built on the $C^{*}$-algebra over the canonical commutation

relations (CCR); see [8]. Specifically, let $\mathcal{H}$ be a complex Hilbert space. Then there is a $C^{*}$-algebra $\mathfrak{A}=\mathfrak{A}(\mathcal{H})$ which is generated by the identity element $\mathbb{1}$ and a family of unitary 
elements $\left\{u_{\xi} \mid \xi \in \mathcal{H} \backslash\{0\}\right\}$ such that

$$
u_{\xi} u_{\eta}=e^{\frac{i}{2} \operatorname{Im}\langle\xi \mid \eta\rangle} u_{\xi+\eta}
$$

for all $\xi, \eta \in \mathcal{H}$, with the understanding that $u_{0}=\mathbb{1}$. Then it follows that there is a unique, completely positive semigroup $\left\{\tau_{t}\right\}_{t \in \mathbb{R}_{+}}$in $\mathfrak{A}$, such that

$$
\tau_{t}\left(u_{\xi}\right)=e^{-t\|\xi\|_{\mathcal{H}}^{2}} u_{\xi} \quad \text { for } \xi \in \mathcal{H} \text {. }
$$

Hence the subalgebra $\mathcal{D} \subset \mathfrak{A}$ spanned by the elements $\left\{u_{\xi} \mid \xi \in \mathcal{H}\right\}$ is contained in the domain of the generator $\delta$, and

$$
\delta\left(u_{\xi}\right)=-\|\xi\|_{\mathcal{H}}^{2} u_{\xi}
$$

It follows from the observation in [3] and [5] that this $\delta$ is completely dissipative with dense domain $\mathcal{D}$ in the $C^{*}$-algebra $\mathfrak{A}$. That is, $\delta$ defined by

$$
\delta(a)=\lim _{t \rightarrow 0_{+}} t^{-1}\left(\tau_{t}(a)-a\right) \quad \text { (norm limit) }
$$

is well defined for $a=u_{\xi} \in \mathcal{D}$, and (IV.1) holds.

We now turn to the general existence problem.

Theorem IV.2. Let $\mathfrak{A}$ be a $C^{*}$-algebra with unit $\mathbb{1}$, and let $\delta$ be a completely dissipative transformation in $\mathfrak{A}$ with dense domain $\mathcal{D}(\delta)$. Assume $\mathbb{1} \in \mathcal{D}(\delta), \delta(\mathbb{1})=0$, and further that $\delta$ is hermitian. Moreover assume that the double conjugate (dual) $\mathfrak{A}^{\prime \prime}$ is an injective $W^{*}$-algebra. Then $\delta$ has an extension $\tilde{\delta}$ to an ultraweakly densely defined transformation in $\mathfrak{A}^{\prime \prime}$ which is at the same time the infinitesimal generator of a completely positive semigroup of normal unital transformations in $\mathfrak{A}^{\prime \prime}$.

We have divided the proof of Theorem IV.2 into two main sections: one is concerned with the analysis of the family of extensions of the partial resolvent operator $(I-\delta)^{-1}$. This analysis leads to a distinguished set of contractive, and maximal, extensions which is associated with a set of extensions $\tilde{\delta}$ of $\delta$. But $\tilde{\delta}$ turns out to be an operator in the enveloping $W^{*}$-algebra of $\mathfrak{A}$. The generation properties of $\tilde{\delta}$ are analyzed in the second section.

\section{EXTENSIONS OF $(I-\delta)^{-1}$}

We may assume that $\delta$ is in fact a closed operator in $\mathfrak{A}$. (If not, it would be possible to replace $\delta$ by the closure $\bar{\delta}$, and $\bar{\delta}$ will have the properties which were listed for $\delta$.) 
This means that the linear space $\mathcal{S}=\operatorname{Ran}(I-\delta)=\{x-\delta(x): x \in \mathcal{D}(\delta)\}$ is closed in $\mathfrak{A}$. In view of the (hermitian) assumption on $\delta$ we note that $\mathcal{S}$ is also selfadjoint, and that $\mathbb{1} \in \mathcal{S}$. The operator $R: \mathcal{S} \rightarrow \mathfrak{A}$ defined by $x-\delta(x) \rightarrow x$, and denoted by $(I-\delta)^{-1}$, is completely positive [2, Prop. 1.2.8]. Clearly $R(\mathbb{1})=\mathbb{1}$.

We now consider the double dual to $\mathfrak{A}$, denoted by $\mathfrak{A}^{\prime \prime}$, as a $W^{*}$-algebra $M$, and make the appropriate identification (via the universal *-representation for $\mathfrak{A}$ ) such that $\mathfrak{A}$ is regarded as a $C^{*}$-subalgebra of $\mathfrak{A}^{\prime \prime}$, and the pre-dual of $\mathfrak{A}^{\prime \prime}$ is identified with the dual $\mathfrak{A}^{\prime}$ of $\mathfrak{A}$. (The reader is referred to $[35, \S 1.17$, p. 42$]$ for details.) Since $M=\mathfrak{A}^{\prime \prime}$ (with the Arens multiplication) is injective as a $W^{*}$-algebra, by the assumption, it follows that a completely positive extension mapping $E: M \rightarrow M$ exists. If we regard $\mathfrak{A}$ as a subalgebra of $M$ (as we shall), then the extension property is given by the identity

$$
R(s)=E(s) \quad \text { for all } s \in \mathcal{S} \text {. }
$$

Note that $\mathcal{S} \subset \mathfrak{A}$, so that $\mathcal{S}$ becomes a subspace of $M$ with the above mentioned identification.

The completely positive transformations of $M$ into itself will be denoted by $C P(M)$, and the space $L(M)$ of completely bounded linear transformations in $M$ gets an ordering arising from the cone $C P(M)$. Indeed, for $F \in L(M)$ we define $E \leq F$ by the requirement that $F-E \in C P(M)$. Among all the particular extensions $F$ of $R, F \in L(M)$, such that $E \leq F$, we choose by Zorn a maximal element $F_{0}$. (For the basic facts on topologies on $C P(M)$ which are needed, the reader is referred to [2, Ch. 1].)

This extension $F_{0}$, described above, has the special property of being $1-1$. We first consider the restriction of $F_{0}$ to the positive elements in $M, M_{+}$, that is. More precisely, we have the implication:

$$
x \in M_{+}, F_{0}(x)=0 \Longrightarrow x=0 .
$$

Let $\eta: M \rightarrow M / \mathcal{S}$ be the canonical linear quotient mapping, and consider the cone $\mathcal{C}$ in the normed quotient space $\mathcal{E}=\mathcal{M} / \mathcal{S}$ given by $\mathcal{C}=\eta\left(M_{+}\right)$.

If the element $x$ in $(\underline{\mathrm{V} .2})$ belongs to $\mathcal{S}$, then the conditions $R(x)=F_{0}(x)=0$ imply $x=0$, since $R=(I-\delta)^{-1}$. Hence, we shall assume that $x$ is not in $\mathcal{S}$. This means that $\eta(x) \in \mathcal{C}$ defines a one-dimensional subspace $\{k \eta(x): k \in \mathbb{C}\}$ in $\mathcal{E}$, and the functional $f: k \eta(x) \rightarrow k$ is nonzero and positive. By Krein's theorem [1, Thm. 1, Ch. 3, p. 157] $f$ extends to a positive 
functional $\tilde{f}$ on $\mathcal{E}$, and we may define

$$
F_{1}(y)=F_{0}(y)+\tilde{f}(\eta(y)) \mathbb{1} \quad \text { for } y \in M
$$

We claim that $F_{1}$ is one of the extensions considered in the Zorn-process which was described above. But $F_{0} \leq F_{1}$, and $F_{0} \neq F_{1}$, contradicting the maximality of $F_{0}$-and so, we must have $x=0$, concluding the proof of $(\overline{V .2})$. (Note that in $(\overline{V .3})$, instead of the identity element $\mathbb{1}$ on the right-hand side of the equation, we could have used any nonzero element in $M_{+}$. The corresponding $F_{1}$-transformation would properly majorize $F_{0}$, and have its range contained in $M$, since the range of $F_{0}$ falls in $M$.)

Since $F_{0}$ is completely positive, we have, in particular, $F_{0}\left(x^{*}\right)=F_{0}(x)^{*}$. So, to establish the identity $N\left(F_{0}\right)=\left\{x \in M: F_{0}(x)=0\right\}=0$, it is enough to show that the hermitian part of $N\left(F_{0}\right)$ is zero. Since we have already considered positive elements, it only remains to consider $x=x^{*} \in N\left(F_{0}\right)$ satisfying $x \notin \mathcal{S}$. Choose a positive real number $k$ such that $x_{k}=x+k \mathbb{1} \in M_{+}$. We then have $F_{0}\left(x_{k}\right)=k$ and $x_{k} \notin \mathcal{S}$. It is possible, therefore, by Krein's theorem, to choose a positive functional $\tilde{f}$ on $\mathcal{E}=M / \mathcal{S}$ satisfying $\tilde{f}\left(\eta\left(x_{k}\right)\right)=l>0$. Then define $F_{2}(y)=F_{0}(y)+\tilde{f}(\eta(y)) \mathbb{1}$ for $y \in M$. It is a simple matter to check that $F_{2}$ is one of the Zorn-extensions. Indeed, $F_{0} \leq F_{2}$ since $\tilde{f}$ is chosen positive. Finally $F_{2}\left(x_{k}\right)=F_{0}\left(x_{k}\right)+l \mathbb{1}>F_{0}\left(x_{k}\right)$. This contradiction to the maximality of $F_{0}$ concludes the proof. Since $N\left(F_{0}\right)=0$, the inverse $F_{0}^{-1}$ is defined on $F_{0}(M)=\left\{F_{0}(x): x \in M\right\}$.

We proceed to show that $F_{0}(M)$ is in fact dense in the $\sigma\left(M, \mathfrak{A}^{\prime}\right)$-topology of $M$ : First note that the extension property (V.1) for $F_{0}$ translates into:

$$
F_{0}(x-\delta(x))=x \quad \text { for } x \in \mathcal{D}(\delta)
$$

and the corresponding transposed mappings in $\mathfrak{A}^{\prime}$ therefore satisfy:

$$
\left(I-\delta^{\prime}\right) F_{0}^{\prime}=I \quad\left(\text { the identity operator in } \mathfrak{A}^{\prime}\right)
$$

Hence $F_{0}^{\prime}$ is $1-1$, and the desired density of $F_{0}(M)$ follows from the bi-polar theorem applied to the $\mathfrak{A}^{\prime}-M$ duality. Note that in fact every extension of $R$ has dense range, because condition (V.5) is satisfied for the most general such extension.

Since $F_{0}$ is an extension of $(I-\delta)^{-1}$ it is clear that $\tilde{\delta}=I-F_{0}^{-1}$ is therefore an extension of $\delta$. 


\section{GENERATION PROPERTIES OF $\tilde{\delta}$}

The operator $\tilde{\delta}$ is closed and densely defined in the $\sigma$-topology of $M$. But $(I-\tilde{\delta})^{-1}=F_{0}$, so we also have $\|x-\tilde{\delta}(x)\| \geq\|x\|$ for all $x \in \mathcal{D}(\tilde{\delta})$. We proceed to show that in fact

$$
\|k x-\tilde{\delta}(x)\| \geq k\|x\|
$$

for all $k>0$ and $x \in \mathcal{D}(\tilde{\delta})$. Indeed, let $\Lambda$ denote the set of $k>0$ such that the inequality (VI.1) is satisfied for all $x \in \mathcal{D}(\tilde{\delta})$. Then we have seen that $k=1$ belongs to $\Lambda$. It turns out that $\Lambda$ is both open and closed as a subset of $\mathbb{R}_{+}$, and our result follows by connectedness.

To show openness, suppose first that $k_{0} \in \Lambda$, and that $k \in \mathbb{R}_{+}$satisfies $\left|k-k_{0}\right|<k_{0}$. We than use (VI.1), for $k_{0}$, in estimating the terms in the Neumann expansion for $(k I-\tilde{\delta})^{-1}$, taken around the point $k_{0}$. Due to the assumption $\left|k-k_{0}\right|<k_{0}$, the Neumann series is convergent, and does indeed define a bounded inverse $R(k, \tilde{\delta})$ to $k I-\tilde{\delta}$. Termwise estimation gives $\|R(k, \tilde{\delta})\| \leq k^{-1}$, and it follows that (VI.1) is satisfied in a neighborhood of $k_{0}$.

Consider next a convergent sequence of points $k_{n} \rightarrow k_{0}$ with $k_{n} \in \Lambda$ and $k_{0} \in \mathbb{R}_{+}$. By assumption the resolvent operators $R\left(k_{n}, \tilde{\delta}\right)=\left(k_{n} I-\tilde{\delta}\right)^{-1}$ exist, and they therefore satisfy the resolvent identity:

$$
R\left(k_{n}, \tilde{\delta}\right)-R\left(k_{m}, \tilde{\delta}\right)=\left(k_{n}-k_{m}\right) R\left(k_{n}, \tilde{\delta}\right) R\left(k_{m}, \tilde{\delta}\right)
$$

as well as the estimate $\left\|R\left(k_{n}, \tilde{\delta}\right)\right\| \leq k_{n}^{-1}$. It follows that the norm-limit $\tilde{R}=\lim _{n} R\left(k_{n}, \tilde{\delta}\right) \in$ $L(M)$ exists, and it is trivial to check that $\tilde{R}$ defines a bounded inverse to $k_{0} I-\tilde{\delta}$. The estimate (VI.1) for $k_{0}$ is now implied in the limit by $\|\tilde{R}\| \leq k_{0}^{-1}$. Hence $\Lambda$ is closed, and the argument is completed.

We have shown that the operator $\tilde{\delta}$ in $M$ is dissipative and closed in the $\sigma\left(M, \mathfrak{A}^{\prime}\right)$ topology. It is, of course, also closed in the norm-topology, and it can be showr that $\mathcal{D}(\tilde{\delta})$ is norm-dense. It follows by semigroup theory [23, 29] that $\tilde{\delta}$ is the infinitesimal generator of a strongly continuous semigroup $\tau_{t}(0 \leq t<\infty)$ of contraction operators in the Banach space $M$.

To show that each $\tau_{t}$ is a normal transformation we consider the adjoint semigroup $\tau_{t}^{\prime}$ (cf. [16]) in the norm-dual $M^{\prime}$ and show that $\tau_{t}^{\prime}$ leaves $\mathfrak{A}^{\prime}$ invariant. Note that $\mathfrak{A}^{\prime}$ is being identified with the predual of the $W^{*}$-algebra $M$, so that we may regard it as a subspace of $M^{\prime}$. 
Let $\tilde{\delta}^{\prime}$ (resp., $F_{0}^{\prime}$ ) denote the transposed operators to $\tilde{\delta}$ (resp., $F_{0}$ ) with respect to the $M-$ $M^{\prime}$ duality. It follows by operator theory that $\tilde{\delta}^{\prime}$ is the generator of $\tau_{t}^{\prime}$, and that $\left(I-\tilde{\delta}^{\prime}\right)^{-1}=$ $F_{0}^{\prime}$. From the construction of $F_{0}$ we now deduce that $\mathfrak{A}^{\prime}$ is invariant under $F_{0}^{\prime}$. Indeed, recall that $\delta^{\prime}$ denotes the transposed transformation to $\delta$ with respect to the $\mathfrak{A}-\mathfrak{A}^{\prime}$ duality. By definition $\mathcal{D}\left(\delta^{\prime}\right)=\left\{a^{\prime} \in \mathfrak{A}^{\prime}: \exists b^{\prime} \in \mathfrak{A}^{\prime},\left\langle b^{\prime}, x\right\rangle=\left\langle a^{\prime}, \delta(x)\right\rangle\right.$ for all $\left.x \in \mathcal{D}(\delta)\right\}$. But for $a^{\prime} \in \mathfrak{A}^{\prime}$ and $x \in \mathcal{D}(\delta)$ we have $\left\langle F_{0}^{\prime}\left(a^{\prime}\right), x-\delta(x)\right\rangle=\left\langle a^{\prime}, x\right\rangle$. Hence, $F_{0}^{\prime}\left(a^{\prime}\right) \in \mathcal{D}\left(\delta^{\prime}\right) \subset \mathfrak{A}^{\prime}$ by (V.5).

An application of the Neumann expansion to $\left(I-\frac{t}{n} \tilde{\delta}^{\prime}\right)^{-1}$ shows that $\mathfrak{A}^{\prime}$ is also invariant under this operator for all $t \geq 0, n \in \mathbb{Z}_{+}$. But $\tau_{t}^{\prime}$ is obtained as a weak*-limit of these operators $(n \rightarrow \infty)$, and the desired invariance $\tau_{t}^{\prime}\left(\mathfrak{A}^{\prime}\right) \subset \mathfrak{A}^{\prime}$ follows.

A final application of the Neumann series, now to the operators $\left(I-\frac{t}{n} \tilde{\delta}\right)^{-1}$, shows that $\tau_{t}$ is completely positive in $M$ for all $t \geq 0$. Indeed $\left(I-\frac{t}{n} \tilde{\delta}\right)^{-1}$ may be expanded in a norm-convergent power series in the completely positive operator $F_{0}=(I-\tilde{\delta})^{-1}$, and $\tau_{t}=$ $\lim _{n \rightarrow \infty}\left(I-\frac{t}{n} \tilde{\delta}\right)^{-1}$.

\section{THE INEQUALITY $\delta\left(x^{*} x\right) \geq \delta(x)^{*} x+x^{*} \delta(x)$}

It was shown in [19] that if $\delta$ is a bounded hermitian linear map in a $C^{*}$-algebra $\mathfrak{A}$, then the following two conditions are equivalent:

$$
e^{t \delta}\left(x^{*} x\right) \geq e^{t \delta}\left(x^{*}\right) e^{t \delta}(x), \quad \forall x \in \mathfrak{A}, t \in \mathbb{R}_{+},
$$

and

$$
\delta\left(x^{*} x\right) \geq \delta\left(x^{*}\right) x+x^{*} \delta(x), \quad \forall x \in \mathfrak{A} .
$$

For unbounded $\mathfrak{A}$ the situation is not as well understood. It is therefore of interest to study the connection between the property (VII.2) for $\delta$, and the other conditions which are customarily used in the applications of unbounded dissipative mappings in operator algebras to quantum dynamics.

Theorem VII.1. Let $\mathfrak{A}$ be a $C^{*}$-algebra with unit $\mathbb{1}$, and let $\delta$ be a completely dissipative transformation in $\mathfrak{A}$ with dense domain $\mathcal{D}(\delta)$. Assume $\mathbb{1} \in \mathcal{D}(\delta)$, and $\delta(\mathbb{1})=0$.

(a) Let $x \in \mathcal{D}(\delta)$ and assume that $x^{*} x \in \mathcal{D}(\delta)$. Then

$$
\delta\left(x^{*} x\right) \geq \delta(x)^{*} x+x^{*} \delta(x)
$$


(b) Suppose both $x$ and $x^{*}$ belong to $\mathcal{D}(\delta)$. Then $\delta\left(x^{*}\right)=\delta(x)^{*}$.

The following results are corollaries to the proofs of Theorems IV.2 and VII.1.

Corollary VII.2. Let $\mathfrak{A}$ be a $C^{*}$-algebra with unit $\mathbb{1}$, and let $\delta$ be completely dissipative in $\mathfrak{A}$ with dense domain $\mathcal{D}(\delta)$, $\mathbb{1} \in \mathcal{D}(\delta), \delta(\mathbb{1})=0$.

(a) If $\mathfrak{A} \subset B(\mathcal{H})$ for some Hilbert space $\mathcal{H}$, then there is a sequence of completely positive maps $E_{n}: \mathfrak{A} \rightarrow B(\mathcal{H}), E_{n}(\mathbb{1})=\mathbb{1}$, such that the following norm-convergence holds:

$$
E_{n}(x) \longrightarrow x \quad \text { for } x \in \mathfrak{A},
$$

and

$$
n\left(E_{n}(x)-x\right) \longrightarrow \delta(x) \quad \text { for } x \in \mathcal{D}(\delta)
$$

(b) If $\mathcal{D}(\delta)$ is hermitian, then $\delta$ is hermitian as well, i.e., $\delta\left(x^{*}\right)=\delta(x)^{*}$ for all $x \in \mathcal{D}(\delta)$, and it is then possible, for each $n$, to choose $E_{n}$ to be 1-1 with dense range.

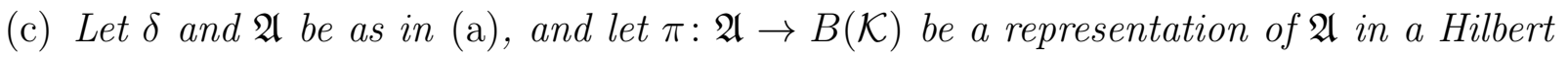
space $\mathcal{K}$. Then there exists a sequence $E_{n} \in C P(\mathfrak{A}, B(\mathcal{K}))$ such that the following norm convergence holds:

$$
E_{n}(x) \longrightarrow \pi(x) \quad \text { for } x \in \mathfrak{A}
$$

and

$$
n\left(E_{n}(x)-\pi(x)\right) \longrightarrow \pi(\delta(x)) \quad \text { for } x \in \mathcal{D}(\delta) .
$$

Proofs. We consider again the range subspace $\mathcal{S}=\operatorname{Ran}(I-\delta)=\{x-\delta(x): x \in \mathcal{D}(\delta)\}$. As in the proof of Theorem $\llbracket .2$ note that $R=(1-\delta)^{-1}: \mathcal{S} \rightarrow \mathfrak{A}$ is completely contractive, and $R(\mathbb{1})=\mathbb{1}$. If $\mathfrak{A}$ is considered as a subalgebra of $B(\mathcal{H})$, where $\mathcal{H}$ is the Hilbert space of the universal representation, then there is, by Arveson's extension theorem [2, Theorem 1.2.9] a completely positive mapping $E: \mathfrak{A} \rightarrow B(\mathcal{H})$ such that

$$
R(s)=E(s) \quad \text { for all } s \in \mathcal{S} \text {. }
$$

If for each $n=1,2, \ldots$ the operator $\delta$ is replaced by $n^{-1} \delta$, then the above argument yields a completely positive map $E_{n}: \mathfrak{A} \rightarrow B(\mathcal{H})$ such that $E_{n}$ is an extension of the partially defined operator $\left(I-n^{-1} \delta\right)^{-1}$. 
We claim that the sequence $\left(E_{n}\right)$ satisfies conditions (i) and (ii) which are listed in Corollary VII.2(公). Indeed, for $x$ in dense $\mathcal{D}(\delta)$ we have $E_{n}\left(x-n^{-1} \delta(x)\right)=x$, and therefore

$$
E_{n}(x)=n^{-1} E_{n}(\delta(x))+x
$$

and

$$
E_{n}(\delta(x))=n\left(E_{n}(x)-x\right)
$$

Passing to the limit in (VII.5), we get (i) for the special case $x \in \mathcal{D}(\delta)$, but then also for all $x$ in $\mathfrak{A}$ by a $3-\varepsilon$ argument since each $E_{n}$ is contractive. The result (ii) of Corollary VII.2(国) is now an immediate consequence of (VII.6).

Returning to the proof of Theorem VII.1, we note that (b) is trivial from (ii). Indeed, for $x$ and $x^{*}$ in $\mathcal{D}(\delta)$ we have

$$
\delta\left(x^{*}\right)=\lim n\left(E_{n}\left(x^{*}\right)-x^{*}\right)=\lim _{n}\left(n\left(E_{n}(x)-x\right)\right)^{*}=\delta(x)^{*} .
$$

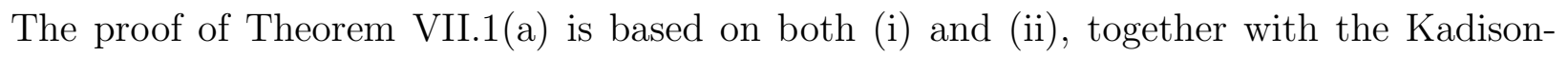
Schwarz inequality for $E_{n}$ : Suppose $x \in \mathcal{D}(\delta)$ and $x^{*} x \in \mathcal{D}(\delta)$. Then $\delta\left(x^{*} x\right)=$ $\lim n\left(E_{n}\left(x^{*} x\right)-x^{*} x\right)$. For each term on the right-hand side we have:

$$
\begin{aligned}
& n\left(E_{n}\left(x^{*} x\right)-x^{*} x\right) \geq n\left(E_{n}(x)^{*} E_{n}(x)-x^{*} x\right) \\
&= \frac{1}{2}\left(\left(n\left(E_{n}(x)-x\right)\right)^{*}\left(E_{n}(x)+x\right)\right. \\
&\left.\quad+\left(E_{n}(x)+x\right)^{*} n\left(E_{n}(x)-x\right)\right) \\
& \longrightarrow \frac{1}{2}\left(\delta(x)^{*}(2 x)+(2 x)^{*} \delta(x)\right)=\delta(x)^{*} x+x^{*} \delta(x),
\end{aligned}
$$

where the last convergence $\longrightarrow$ is based on (i) and (ii) from Corollary VII.2(国). Since $\delta\left(x^{*} x\right)$ is obtained in the limit on the left, the desired inequality (VII.3) in (a) of Theorem VII.1 follows.

Only part (b) of the corollary remains. The technique from the proof of Theorem IV.2 is applied here. We go back to the extension $E$ from (VII.4) in the beginning of the present proof. Consider the ordering on all the extensions $F$ of $R, F \in L(\mathfrak{A}, B(\mathcal{H}))$, which is induced by the cone $C P(\mathfrak{A}, B(\mathcal{H}))$, and choose by Zorn a particular extension $F, E \leq F$, which is maximal. The argument from the proof of Theorem IV.2 then shows that $F$ is $1-1$, and the range $\operatorname{Ran}(F)$ is dense. It follows that the operator $\tilde{\delta}=I-F^{-1}: \operatorname{Ran}(F) \rightarrow \mathfrak{A}$ exists and satisfies $\tilde{\delta}(x)=\delta(x)$ for all $x \in \mathcal{D}(\delta)$. 
If $\alpha$ is a positive real number, then the same construction may be carried out for the transformation $\alpha \delta$, instead of $\delta$. Hence we get completely positive unital maps $F_{\alpha}$ such that the inverse $F_{\alpha}^{-1}$ exists for each $\alpha$, and the domain of $I-F_{\alpha}^{-1}$ contains $\mathcal{D}(\delta)$. Moreover $\tilde{\delta}_{\alpha}=I-F_{\alpha}^{-1}$ satisfies $\tilde{\delta}_{\alpha}(x)=\delta(x)$ for $x \in \mathcal{D}(\delta)$. To get a sequence of mappings satisfying the conditions in Corollary $\nabla \Pi .2(\mathrm{~b})$, we need only take $E_{n}=F_{n^{-1}}$ in the special case $\alpha=n^{-1}$.

The proof of part (드) in the corollary is parallel to (国) with the following modification: Arveson's extension theorem is now applied to the mapping $\pi \circ(I-\delta)^{-1}: \mathcal{S} \rightarrow B(\mathcal{K})$.

\section{THE IMPLEMENTATION PROBLEM}

The conclusion (ii') in Corollary VII.2(ㄷ) is of interest when one wants to implement the transformation $\delta$ by a dissipative operator in Hilbert space. In particular, one is interested in implementing a completely dissipative $\delta$-operator by a dissipative Hilbert-space operator. We shall establish a clear two-way connection between the dissipative notion for $\delta$, and for the implementing Hilbert-space operator.

Theorem VIII.1. Let $\mathfrak{A}$ be a $C^{*}$-algebra with unit $\mathbb{1}$, and let $\delta$ be a completely dissipative transformation in $\mathfrak{A}$ with dense domain $\mathcal{D}(\delta)$. Assume $\mathbb{1} \in \mathcal{D}(\delta)$ and $\delta(\mathbb{1})=0$. Let $\omega$ be a state of $\mathfrak{A}$, and let $\left(\pi_{\omega}, \mathcal{K}_{\omega}, \Omega\right)$ be the corresponding GNS representation of $\mathfrak{A}$. Let $\tilde{\omega}$ be the vector state on $B\left(\mathcal{K}_{\omega}\right)$ given by the cyclic vector $\Omega$, i.e., $\tilde{\omega}(X)=\langle X \Omega \mid \Omega\rangle$ for $X \in B\left(\mathcal{K}_{\omega}\right)$, and assume that it is possible to choose the sequence $\left(E_{n}\right) \subset C P\left(\mathfrak{A}, B\left(\mathcal{K}_{\omega}\right)\right)$ from Corollary VII.2(C) in such a manner that

$$
\tilde{\omega}\left(E_{n}(x)\right)=\omega(x) \quad \text { for all } x \in \mathfrak{A} \text {. }
$$

Then there is a dissipative operator $L_{\omega}$ in $\mathcal{K}_{\omega}$ such that

$$
\pi_{\omega}(\delta(x)) \Omega=L_{\omega}\left(\pi_{\omega}(x) \Omega\right) \quad \text { for all } x \in \mathcal{D}(\delta)
$$

Proof. Let $\pi=\pi_{\omega}, \mathcal{K}=\mathcal{K}_{\omega}$, and let $\left(E_{n}\right) \subset C P(\mathfrak{A}, B(\mathcal{K}))$ be a sequence which, along with the conditions listed in Corollary VII.2(c), also fulfills the invariance restriction (VIII.1) of the present theorem. For each $n$ define an operator $C_{n}$ in $\mathcal{K}$ as follows:

$$
C_{n}(\pi(x) \Omega)=E_{n}(x) \Omega, \quad x \in \mathfrak{A}
$$


Then

$$
\begin{aligned}
\left\|C_{n} \pi(x) \Omega\right\|^{2} & =\left\|E_{n}(x) \Omega\right\|^{2}=\tilde{\omega}\left(E_{n}(x)^{*} E_{n}(x)\right) \\
& \leq \tilde{\omega}\left(E_{n}\left(x^{*} x\right)\right)=\omega\left(x^{*} x\right)=\left\langle\pi\left(x^{*} x\right) \Omega \mid \Omega\right\rangle=\|\pi(x) \Omega\|^{2},
\end{aligned}
$$

where the norm is that of $\mathcal{K}$, and where the Schwarz inequality is applied to $E_{n}$. It follows that $C_{n}$ is well defined, and that it extends by limits (in $\mathcal{K}$ ) to a contraction operator, $C_{n} \in B(\mathcal{K}),\left\|C_{n}\right\| \leq 1$.

By Corollary VII.2(C) (ii'), we then have

$$
\begin{aligned}
\pi(\delta(x)) \Omega & =\lim n\left(E_{n}(x) \Omega-\pi(x) \Omega\right) \\
& =\lim n\left(C_{n}(\pi(x) \Omega)-\pi(x) \Omega\right) \\
& =\lim n\left(C_{n}-I\right) \pi(x) \Omega \quad \text { for } x \in \mathcal{D}(\delta)
\end{aligned}
$$

As a consequence, the following quadratic form on $\mathcal{K}$ :

$$
\pi(x) \Omega, \pi(y) \Omega \longrightarrow \lim \left\langle n\left(C_{n}-I\right) \pi(x) \Omega \mid \pi(y) \Omega\right\rangle_{\mathcal{K}}
$$

is well defined. Using the contractive property of $C_{n}$, it is easy to show that this quadratic form is given by a dissipative operator $L$; that is to say

$$
\lim \left\langle n\left(C_{n}-I\right) \pi(x) \Omega \mid \pi(y) \Omega\right\rangle=\langle L \pi(x) \Omega \mid \pi(y) \Omega\rangle
$$

Since the limit on the left is also equal to the inner product

$$
\langle\pi(\delta(x)) \Omega \mid \pi(y) \Omega\rangle
$$

the identity (VIII.2) of the theorem follows.

\section{A CONDITION FOR COMPLETE DISSIPATIVENESS}

In applications [18, 24, 33] it is often possible to determine the derivation $\delta$ in a particular representation. If moreover the derivation is known to be implemented by a dissipative operator in the corresponding Hilbert space, then it follows in special cases that $\delta$ itself is completely dissipative.

Theorem IX.1. Let $\mathfrak{A}$ be a $C^{*}$-algebra with unit $\mathbb{1}$ and let $\delta$ be a densely defined transformation in $\mathfrak{A}$ such that $\mathbb{1} \in \mathcal{D}(\delta)$ and $\delta(\mathbb{1})=0$. Let $\omega$ be a state on $\mathfrak{A}$ such that $\delta$ is implemented 
by a dissipative Hilbert-space operator $L$ in the representation $\pi_{\omega}$. Assume moreover that $\pi_{\omega}$ is faithful, and that $L \Omega=0$, where $\Omega$ denotes the cyclic vector in the GNS representation. Then $\delta$ is completely dissipative on its domain.

Proof. Let $\mathcal{H}=\mathcal{H}_{\omega}$ be the Hilbert space of the faithful representation $\pi_{\omega}$ and let $L$ be the operator in $\mathcal{H}$ which is assumed to exist, satisfying conditions (ii) and (iil) below:

(i) The domain of $L$ is $\pi_{\omega}(\mathcal{D}(\delta)) \Omega$, and $L$ is a dissipative operator in the Hilbert space $\mathcal{H}$;

(ii) $L$ implements $\delta$ in the representation $\pi_{\omega}$, which is equivalent to the requirement that $L^{*}$ is defined on $\pi_{\omega}(\mathcal{D}(\delta)) \Omega$, and that on this domain the following operator identity is valid:

$$
\pi(\delta(a))=L \pi(a)+\pi(a) L^{*} \quad \text { for all } a \in \mathcal{D}(\delta) .
$$

We show first that $\delta$ must necessarily be a dissipative operator. Indeed, by Phillips's theorem [30, Thm. 1.1.3] an extension $\tilde{L}$ of $L$ exists which is the infinitesimal generator of a strongly continuous semigroup $S(t)$ of contraction operators in the Hilbert space $\mathcal{H}$. We note that $S(t)$ implements a semigroup $\sigma(t)$ of positive mappings in $B(\mathcal{H})$, given by

$$
\sigma(t)(A)=S(t) A S(t)^{*}
$$

for all $t \in[0, \infty)$ and $A \in B(\mathcal{H})$. By semigroup theory we note that the generator ( $\zeta$ say) of $\sigma(t)$ is dissipative, so the following estimate holds:

$$
\|A-\alpha \zeta(A)\| \geq\|A\|
$$

for all $\alpha \in[0, \infty)$ and $A \in \mathcal{D}(\zeta)$.

If $\delta_{\omega}$ denotes the operator $\pi_{\omega}(a) \rightarrow \pi_{\omega}(\delta(a))$ with domain $\pi_{\omega}(\mathcal{D}(\delta)$ ), then we claim (easy proof) that

$$
\delta_{\omega}(A)=\zeta(A) \quad \text { for all } A \in \mathcal{D}\left(\delta_{\omega}\right)
$$

and the known estimate (IX.3) above then implies

$$
\left\|\pi_{\omega}(a)-\alpha \pi_{\omega}(\delta(a))\right\| \geq\left\|\pi_{\omega}(a)\right\|
$$

for $a \in \mathcal{D}(\delta)$ and $\alpha \in[0, \infty)$. But $\pi_{\omega}$ is faithful (and hence isometric), so (IX.5) is in fact equivalent to the dissipation estimate

$$
\|a-\alpha \delta(a)\| \geq\|a\|
$$


for the operator $\delta$ itself.

For each $n=1,2, \ldots$, we now consider the tensor-product construction of the $C^{*}$-algebra $\mathfrak{A}$ with the $n$-by- $n$ complex matrices $M_{n}$; and we define $\mathfrak{A}_{n}=\mathfrak{A} \otimes M_{n}, \delta_{n}=\delta \otimes \mathrm{id}_{n}$, the operator obtained by application of $\delta$ to each entry $a_{i j}$ in the matrix representation of elements in $\mathfrak{A}_{n}, \omega_{n}=\omega \otimes \operatorname{tr}_{n}$ where $\operatorname{tr}_{n}$ denotes the normalized trace on $M_{n}, \pi_{\omega_{n}}$ : the GNS representation of $\mathfrak{A}_{n}$ associated to $\omega_{n}$.

The problem is to show that each of the operators $\delta_{n}$ is dissipative. We show that in fact $\delta_{n}$ is implemented by a dissipative Hilbert-space operator in the representation $\pi_{\omega_{n}}$. Hence, the first part of the proof applies and yields the conclusion of the claim since each representation $\pi_{\omega_{n}}$ is faithful, being the tensor product of faithful representations.

Let $\mathcal{H}_{n}$ denote the representation Hilbert space of $\pi_{\omega_{n}}$. We proceed to find a dissipative operator $L_{n}$ in $\mathcal{H}_{n}$ such that $\delta_{n}$ is implemented by $L_{n}$. In view of (IX.1) this means that

$$
\pi_{\omega_{n}}\left(\delta_{n}(a)\right)=L_{n} \pi_{\omega_{n}}(a)+\pi_{\omega_{n}}(a) L_{n}^{*}
$$

for all $a \in \mathcal{D}\left(\delta_{n}\right)=\mathcal{D}\left(\delta_{n}\right) \otimes M_{n}$ (algebraic tensor product) $\subset \mathfrak{A}_{n}$ as an operator identity on $\pi_{\omega_{n}}\left(\mathcal{D}\left(\delta_{n}\right)\right) \Omega_{n} \subset \mathcal{H}_{n}$. Here $\Omega_{n}$ denotes the cyclic vector for the representation $\pi_{\omega_{n}}$, i.e.,

$$
\omega_{n}(a)=\left\langle\pi_{\omega_{n}}(a) \Omega_{n} \mid \Omega_{n}\right\rangle \quad \text { for all } a \in \mathfrak{A}_{n} .
$$

Our next step is the verification of the following:

$$
\begin{aligned}
& \operatorname{Re} \omega_{n}\left(a^{*} \delta_{n}(a)\right) \leq 0 \quad \text { for all } a \in \mathcal{D}\left(\delta_{n}\right), \\
& L_{n} \Omega_{n}=0, \\
& \omega_{n}\left(a^{*} \delta_{n}(a)\right)=\left\langle L_{n} \pi_{\omega_{n}}(a) \Omega_{n} \mid \pi_{\omega_{n}}(a) \Omega_{n}\right\rangle \quad \text { for } a \in \mathcal{D}\left(\delta_{n}\right) .
\end{aligned}
$$

It will follow from (IX.7) and (IX.9) that an implementing operator $L_{n}$ satisfying (IX.8) must necessarily be dissipative.

Note that (IX.8) is verified for $n=1$ by assumption. Hence $\omega\left(a^{*} \delta(a)\right)=$ $\langle\pi(\delta(a)) \Omega \mid \pi(a) \Omega\rangle=\left\langle L \pi(a) \Omega+\pi(a) L^{*} \Omega \mid \pi(a) \Omega\right\rangle$. Substitution of $L^{*} \Omega=-L \Omega=0$ into this identity yields identity (IX.9) for the case $n=1$.

Let $T_{n}$ denote the trace-vector for the trace representative $\tau_{n}$ of $M_{n}$. Then $\pi_{\omega_{n}}=\pi \otimes \tau_{n}$, and therefore

$$
\begin{aligned}
\left\langle\pi_{\omega_{n}}(a \otimes b) \Omega \otimes T_{n} \mid \Omega \otimes T_{n}\right\rangle & =\left\langle\pi(a) \Omega \otimes \tau_{n}(b) T_{n} \mid \Omega \otimes T_{n}\right\rangle \\
& =\langle\pi(a) \Omega \mid \Omega\rangle\left\langle\tau_{n}(b) T_{n} \mid T_{n}\right\rangle=\omega(a) \operatorname{tr}_{n}(b) \\
& =\omega \otimes \operatorname{tr}_{n}(a \otimes b)=\omega_{n}(a \otimes b)
\end{aligned}
$$


for all $a \in \mathfrak{A}$ and $b \in M_{n}$. Hence $\Omega_{n}=\Omega \otimes T_{n}$. If we can show that a simple tensor operator $L_{n}$ implements $\delta_{n}$ in $\pi_{\omega_{n}}$, then identity (IX.9), for arbitrary $n$, follows from the case $n=1$ which was established above.

However, it is easy to see that the operator $L_{n}=L \otimes I_{n}$ satisfies the requirements which were listed above. Indeed

$$
\begin{aligned}
\pi_{\omega_{n}}\left(\delta_{n}(a \otimes b)\right) & =\pi_{\omega}(\delta(a)) \otimes \tau_{n}(b) \\
& =\left(L \pi_{\omega}(a)+\pi_{\omega}(a) L^{*}\right) \otimes \tau_{n}(b) \\
& =L_{n} \pi_{\omega}(a) \otimes \tau_{n}(b)+\pi_{\omega}(a) \otimes \tau_{n}(b) L_{n}^{*} \\
& =L_{n} \pi_{\omega_{n}}(a \otimes b)+\pi_{\omega_{n}}(a \otimes b) L_{n}^{*}
\end{aligned}
$$

for all $a \in \mathfrak{A}$ and $b \in M_{n}$. It follows that $L_{n}$ implements $\delta_{n}$ in $\pi_{\omega_{n}}$.

Only the verification of (IX.7) for $n>1$ then remains. Let $a_{i j} \in \mathfrak{A}$ be the matrix entries in some $a \in \mathfrak{A}_{n}=\mathfrak{A} \otimes M_{n}$. Then the $(i, j)^{\prime}$ th entry $c_{i j}$ in $a^{*} \delta_{n}(a)$ is $\sum_{k=1}^{n} a_{k i}^{*} \delta\left(a_{k j}\right)$. Hence

$$
\begin{aligned}
\omega_{n}\left(a^{*} \delta_{n}(a)\right) & =\left(\omega \otimes \operatorname{tr}_{n}\right)\left(c_{i j}\right) \\
& =\sum_{i=1}^{n} \omega\left(c_{i i}\right)=\sum_{i} \sum_{k} \omega\left(a_{k i}^{*} \delta\left(a_{k i}\right)\right) .
\end{aligned}
$$

Since $\operatorname{Re} \omega\left(a_{k i}^{*} \delta\left(a_{k i}\right)\right) \leq 0$, (IX.7) follows.

Remark IX.2. In the foundations of irreversible statistical thermodynamics [14, 20, 21, 24, 28], the most conclusive results have been obtained for dynamical semigroups which are described mathematically as strongly continuous, completely positive, contraction semigroups $T_{t}$ on the Banach space $\mathcal{T}(\mathcal{H})$ of all trace-class operators on a given separable $\infty$-dimensional Hilbert space $\mathcal{H}$. Lindblad [28] found a formula for the infinitesimal generator

$$
W=\left.\frac{d}{d t} T_{t}\right|_{t=0}
$$

in the case of norm-continuous semigroups, and Davies [15] extended the results to strongly continuous $T_{t}$ (i.e., unbounded generator $W$ ), satisfying certain side conditions. The condition of relevance to our paper is the invariance assumption of [15] that

$$
T_{t}^{\prime}(\mathcal{C}(\mathcal{H})) \subset \mathcal{C}(\mathcal{H})
$$

for all $t \in[0, \infty)$, where $\mathcal{C}(\mathcal{H})$ denotes the compact operators, and $T_{t}^{\prime}$ the conjugate semigroup on $B(\mathcal{H})$. Our Theorem $I \nabla .2$ does not apply to the algebra $\mathfrak{A}=B(\mathcal{H})$ since $B(\mathcal{H})^{\prime \prime}$ is known not to be injective [11]. (Of course, $B(\mathcal{H})$ is injective by Arveson's theorem.) 
However, Theorem IV.2 combined with the above results suggests that a $W^{*}$-algebra, properly smaller than $B(\mathcal{H})$, is suitable for quantum dynamics. On the one hand, $B(\mathcal{H})$ (or $\mathcal{T}(\mathcal{H})$ in the conjugate (dual) formulation) is too big to accomodate the extensions; and, on the other hand, the requirement that $\mathcal{C}(\mathcal{H})$ contain the domain of the generator also appears to be too restrictive.

\section{UNBOUNDED *-DERIVATIONS}

Let $\mathfrak{A}$ be a unital $C^{*}$-algebra, and let $\mathcal{D}(\delta)$ be a dense *-subalgebra containing the identity 1. A linear transformation $\delta: \mathcal{D}(\delta) \rightarrow \mathfrak{A}$ is said to be a (unbounded) *-derivation if $\delta(a b)=$ $\delta(a) b+a \delta(b)$ for $a, b \in \mathcal{D}(\delta)$, and $\delta\left(a^{*}\right)=\delta(a)^{*}$ for $a \in \mathcal{D}(\delta)$.

Since, for *-derivations, one is primarily interested in extensions which are also *derivations, it is natural to work with a two-sided condition in place of the dissipative notions which were studied in the previous sections for more general operators. The following such two-sided condition was suggested by Sakai [36], and adopted by several authors in subsequent research on unbounded $*$-derivations.

Definition X.1. A $*$-derivation $\delta: \mathcal{D}(\delta) \rightarrow \mathfrak{A}$ is said to be well behaved if for all positive $a \in \mathcal{D}(\delta)$ there is a state $\phi$ on $\mathfrak{A}$ such that $\phi(a)=\|a\|$ and $\phi(\delta(a))=0$.

The argument in the previous section yields:

Proposition X.2. Let $\delta: \mathcal{D}(\delta) \rightarrow \mathfrak{A}$ be a $*$-derivation. Then the following four conditions are equivalent:

(i) $\delta$ is well behaved.

(ii) For all positive $a \in \mathcal{D}(\delta)$, and for all states $\phi$ on $\mathfrak{A}$ satisfying $\phi(a)=\|$ a $\|$, we have $\phi(\delta(a))=0$.

(iii) Each of the operators $\pm \delta$ is dissipative.

(iv) $\|a+\alpha \delta(a)\| \geq\|a\|$ for all $\alpha \in \mathbb{R}$ and all $a \in \mathcal{D}(\delta)$.

Definition X.3. A *-derivation $\delta: \mathcal{D}(\delta) \rightarrow \mathfrak{A}$ is said to be well behaved in the matricial sense if, for each $n=1,2, \ldots$, the $*$-derivation $\delta_{n}=\delta \otimes \operatorname{id}_{n}: \mathcal{D}(\delta) \otimes M_{n} \rightarrow \mathfrak{A} \otimes M_{n}$ is well behaved. Recall that $\delta_{n}$ may be regarded as a transformation on $n$-by- $n$ matrices with entries in $\mathfrak{A}$. For such a matrix $a=\left(a_{i j}\right), i, j=1, \ldots, n$, we have $\delta_{n}(a)=\left(\delta\left(a_{i j}\right)\right)$. 
Theorem X.4. Every well-behaved *-derivation is also well behaved in the matricial sense (i.e., completely well behaved).

Lemma X.5. Let $\delta: \mathcal{D}(\delta) \rightarrow \mathfrak{A}$ be a well-behaved $*$-derivation, and let $a \in \mathcal{D}(\delta)$ be positive. Then there is a state $\phi$ on $\mathfrak{A}$ such that $\phi(a)=\|a\|$, and $\phi(\delta(b))=0$ for a dense set of elements $b \in C^{*}(a) \cap \mathcal{D}(\delta)$. (Here $C^{*}(a)$ denotes the abelian $C^{*}$-subalgebra generated by $a$; and every element in $C^{*}(a)$ can be approximated in norm by a sequence of elements $b$ satisfying the conclusion of the lemma.)

Proofs. The implication (ii) $\Rightarrow$ (iii) in Proposition X.2 is the key to the proof of Lemma X.5. Since functional calculus is also applied, we shall assume in fact that $\delta$ is closed. By a result of Kishimoto-Sakai [36] this is no loss of generality. Let a be a positive element in $\mathcal{D}(\delta)$. Note that the Gelfand-transform sets up an isomorphism between the $C^{*}$-algebras $C^{*}(a)$ and $C(\operatorname{sp}(a))$, continuous functions on the spectrum of $a$. Let $\lambda_{0}=1 . u \cdot b \cdot \operatorname{sp}(a)$. Then the state $c \rightarrow c\left(\lambda_{0}\right)$ on $C(\operatorname{sp}(a))$ corresponds to a state on $C^{*}(a)$ via the Gelfand-transform. The latter state is then extended to $\mathfrak{A}$ by Krein's theorem, and the extended state is denoted by $\phi$. It has the multiplicative property: $\phi\left(b_{1} b_{2}\right)=\phi\left(b_{1}\right) \phi\left(b_{2}\right)$ for $b_{1}, b_{2} \in C^{*}(a)$.

Now let $g$ be a non-decreasing (monotone) continuous real function defined on $\operatorname{sp}(a)$. Then the Gelfand-transform of $g(a)$ achieves its maximum at the point $\lambda_{0}$ since the transform of $a$ does. But it is known that if $g$ is also of class $C^{2}$ (two continuous derivatives) then $g(a) \in \mathcal{D}(\delta) \cap C^{*}(a)$. Hence $\phi(g(a))=\|g(a)\|$. An application of Proposition X.2, (iil) $\Rightarrow$ (iii), then yields the conclusion

$$
\phi(\delta(g(a)))=0
$$

The restriction of an arbitrary monomial $\lambda^{n}$ to $\operatorname{sp}(a)$ satisfies the conditions listed for $g$. Hence, by Stone-Weierstrass there is a dense set of elements $b \in C^{*}(a) \cap \mathcal{D}(\delta)$ satisfying the conclusion of the lemma. (Alternatively, every positive function $f$ in $C^{4}$ may be written in the form $f=g_{1}-g_{2}$, with $g_{1}$ and $g_{2}$ both having the properties listed above for $g$, we conclude that $\phi(\delta(f(a)))=\phi\left(\delta\left(g_{1}(a)\right)\right)-\phi\left(\delta\left(g_{2}(a)\right)\right)=0$.)

Now, for each fixed element $a \in \mathcal{D}(\delta)_{+}$we choose a state $\phi=\phi_{a}$ and a dense $*$-subalgebra $\mathfrak{B}=\mathfrak{B}_{a}$ of $C^{*}(a)$ according to Lemma X.5 i.e., we require that $\phi_{a}(\delta(b))=0$ for $b \in \mathfrak{B}_{a}$, as well as $\phi_{a}(a)=\|a\|$. Consider the GNS representation of the algebra $\mathfrak{B}$, resp. $\mathfrak{A}$, with representation space $\mathcal{H}_{\phi}$, resp. $\mathcal{K}_{\phi}$, and define:

$$
\mathcal{H}=\sum^{\otimes} \mathcal{H}_{\phi}, \quad \text { resp. }, \quad \mathcal{K}=\sum^{\otimes} \mathcal{K}_{\phi} .
$$


Then $\mathcal{H}$ is a closed subspace of the Hilbert space $\mathcal{K}$, and we can then define an operator $S$ with dense domain from $\mathcal{H}$ to $\mathcal{K}$ as follows:

$$
S \pi_{\phi}(b) \Omega_{\phi}=\pi_{\phi}(\delta(b)) \Omega_{\phi} \quad \text { for } b \in \mathfrak{B}_{\phi} .
$$

For vectors $\xi_{1}$ and $\xi_{2}$ in the domain of $S$ we have

$$
\left\langle S \xi_{1} \mid \xi_{2}\right\rangle+\left\langle\xi_{1} \mid S \xi_{2}\right\rangle=0
$$

The verification of (X.3) may be based on the direct-sum decomposition (X.1) above. If $\xi_{i}=\sum_{\phi}^{\otimes} \pi\left(b_{i}\right) \Omega_{\phi}$ for $i=1,2$ and $b_{i} \in \mathfrak{B}_{\phi}$, then identity (X.3) reduces to

$$
\sum\left\langle\pi_{\phi}\left(\delta\left(b_{1}\right)\right) \Omega_{\phi} \mid \pi_{\phi}\left(b_{2}\right) \Omega_{\phi}\right\rangle+\sum\left\langle\pi_{\phi}\left(b_{1}\right) \Omega_{\phi} \mid \pi_{\phi}\left(\delta\left(b_{2}\right)\right) \Omega_{\phi}\right\rangle=0
$$

The individual terms work out to be:

$$
\phi\left(b_{2}^{*} \delta\left(b_{1}\right)\right)+\phi\left(\delta\left(b_{2}\right)^{*} b_{1}\right)=\phi\left(\delta\left(b_{2}^{*} b_{1}\right)\right)=0 .
$$

Hence, the symmetry condition (X.3 is hereby reduced to the conclusion of Lemma X.5 for a given well-behaved derivation $\delta$.

If $P$ denotes the orthogonal projection in $\mathcal{K}$ with range $\mathcal{H}$, identity (X.3) implies that the operator $\xi \rightarrow P S \xi$ may in fact be regarded as a skew symmetric operator in the Hilbert space $\mathcal{H}$, with dense domain there. We shall also denote this operator by $S$. The verification of the identity

$$
\pi(\delta(b))=S \pi(b)-\pi(b) S
$$

is left to the reader.

Following the idea of $₫ \mathrm{IX}$, we now consider the $*$-derivations $\delta_{n}=\delta \otimes \mathrm{id}_{n}$ (for each $n=1,2, \ldots$ ) introduced in Definition X.3. For a given $*$-algebra $\mathfrak{C}$ we denote by $\mathfrak{C}_{n}$ the *-algebra $\mathfrak{C} \otimes M_{n}$. Correspondingly, *-algebras $\mathcal{D}(\delta)_{n}, \mathfrak{A}_{n}$, and $\mathfrak{B}_{n}$ are defined for each $n$. Application of the GNS representation to each $\phi_{n}=\phi \otimes \operatorname{tr}_{n}$ yields sequences of Hilbert spaces

$$
\mathcal{H}^{(n)} \subset \mathcal{K}^{(n)}
$$

as in w.1 with each $\mathcal{H}^{(n)}$, resp., $\mathcal{K}^{(n)}$, a direct sum of GNS representation spaces associated to $\phi_{n}$.

The calculations in $₫ \mathbb{I X}$ show that the operator $S_{n}=S \otimes I_{n}$ satisfies the $n$ 'th-order version of (X.2), that is, (X.2) holds with the quadruple $S, \pi, \mathfrak{B}, \delta$ replaced by $S_{n}, \pi_{n}, \mathfrak{B}_{n}, \delta_{n}$. 
Similarly $\left\langle S_{n} \xi_{1}^{(n)} \mid \xi_{2}^{(n)}\right\rangle+\left\langle\xi_{1}^{(n)} \mid S_{n} \xi_{2}^{(n)}\right\rangle=0$ for vectors $\xi_{i}^{(n)}, i=1,2$, in the respective domains.

Hence Theorem IX.1 in $₫ \mathrm{IX}$ implies that each of the operators $\pm \delta_{n}$ for $n=1,2, \ldots$ is dissipative. By Proposition X.2 (iii) $\Rightarrow$ (ii), it follows that $\delta_{n}$ is well behaved, concluding the proof of Theorem X.4.

As an application of the theorem we get the following existence result for generator extensions of well-behaved $*$-derivations $\delta: \mathcal{D}(\delta) \rightarrow \mathfrak{A}$ in nuclear $C^{*}$-algebras $\mathfrak{A}$. Indeed, if $\delta$ is such a $*$-derivation, each of the operators $\pm \delta$ is completely dissipative. Hence, by Theorem

IV.2 there are extensions $\tilde{\delta}_{ \pm} \supset \pm \delta$ to infinitesimal generators of dynamical semigroups $a_{t}^{( \pm)}$ in the enveloping $W^{*}$-algebra $\mathfrak{A}^{\prime \prime}$.

\section{Acknowledgments}

We thank William Arveson for a helpful email exchange concerning the existence problem for completely positive semigroups. The book [6] offers a different approach. We are grateful to Brian Treadway for putting a first draft with rewrites and revisions into beautiful $\mathrm{T}_{\mathrm{EX}}$.

[1] Ahiezer, N.I., and Krein, M., Some Questions in the Theory of Moments, Transl. Math. Monographs, Vol. 2, American Mathematical Society, Providence, 1962.

[2] Arveson, W., Subalgebras of $C^{*}$-algebras, Acta Math. 123 (1969), 141-224.

[3] Arveson, W., Dynamical invariants for noncommutative flows, Operator Algebras and Quantum Field Theory: Proceedings of the conference held at Accademia Nazionale dei Lincei, Roma, Italy, July 1-6, 1996 (S. Doplicher, R. Longo, J.E. Roberts, and L. Zsido, eds.), International Press, Cambridge, MA, 1997, pp. 476-514.

[4] Arveson, W., The domain algebra of a CP-semigroup, Pacific J. Math. 203 (2002), 67-77.

[5] Arveson, W., The heat flow of the CCR algebra, Bull. London Math. Soc. 34 (2002), 73-83.

[6] Arveson, W., Noncommutative Dynamics and E-Semigroups, Springer Monographs in Mathematics, Springer-Verlag, New York, 2003.

[7] Batty, C.J.K., Dissipative mappings with approximately invariant subspaces, J. Funct. Anal. 32 (1979), 336-341. 
[8] Bhat, B.V.R., Cocycles of CCR Flows, Mem. Amer. Math. Soc. 149 (2001), no. 709.

[9] Bratteli, O., and Robinson, D.W., Operator Algebras and Quantum Statistical Mechanics, 2nd ed., vol. II, Springer-Verlag, Berlin-New York, 1996.

[10] Bratteli, O., and Kishimoto, A., Generation of semigroups, and two-dimensional quantum lattice systems, J. Funct. Anal. 35 (1980), 344-368.

[11] Choi, M.-D., and Effros, E., Injectivity and operator spaces, J. Funct. Anal. 24 (1977), 156209.

[12] Choi, M.-D., and Effros, E., Nuclear $C^{*}$-algebras and injectivity: the general case, Indiana Univ. Math. J. 26 (1977), 443-446.

[13] Connes, A., Classification of injective factors: Cases $\mathrm{II}_{1}, \mathrm{II}_{\infty}, \mathrm{III}_{\lambda}, \lambda \neq 1$, Ann. of Math. (2) 104 (1976), 73-115.

[14] Davies, E.B., Quantum Theory of Open Systems, Academic Press, London-New York, 1976.

[15] Davies, E.B., Generators of dynamical semigroups, J. Funct. Anal. 34 (1979), 421-432.

[16] de Leeuw, K., On the adjoint semigroup and some problems in the theory of approximation. Math. Z. 73 (1960), 219-234.

[17] Dubin, D.A., and Sewell, G.L., Time translations in the algebraic formulation of statistical mechanics, J. Math. Phys. 11 (1970), 2990-2998.

[18] Emch, G.G., Algebraic Methods in Statistical Mechanics and Quantum Field Theory, WileyInterscience, New York, 1972.

[19] Evans, D.E., and Hanche-Olsen, H., The generators of positive semigroups, J. Funct. Anal. 32 (1979), 207-212.

[20] Evans, D.E., and Lewis, J.T., Dilations of Irreversible Evolutions in Algebraic Quantum Theory, Comm. Dublin Inst. Adv. Studies Ser. A No. 24 (1977).

[21] Evans, D.E., A review on semigroups of completely positive maps, Mathematical Problems in Theoretical Physics (Lausanne, 1979) (K. Osterwalder, ed.), Lecture Notes in Physics, vol. 116, Springer-Verlag, Berlin-Heidelberg-New York, 1980, pp. 400-406.

[22] Ferrari, P.A., Maes, C., Ramos, L., and Redig, F., On the hydrodynamic equilibrium of a rod in a lattice fluid, J. Phys. A 33 (2000), 4725-4740.

[23] Hille, E., and Phillips, R.S., Functional Analysis and Semi-groups, American Mathematical Society, Providence, 1957.

[24] Ingarden, R.S., and Kossakowski, A., On the connection of nonequilibrium information the- 
ormodynamics with non-Hamiltonian quantum mechanics of open systems, Ann. Physics 89 (1975), 451-485.

[25] Jakšić, V., and Pillet, C.-A., On entropy production in quantum statistical mechanics, Comm. Math. Phys. 217 (2001), 285-293.

[26] Jorgensen, P.E.T., Approximately invariant subspaces for unbounded linear operators, II, Math. Ann. 227 (1977), 177-182.

[27] Lindblad, G., Cloning the quantum oscillator, J. Phys. A 33 (2000), 5059-5076.

[28] Lindblad, G., On the generators of quantum dynamical semigroups, Comm. Math. Phys. 48 (1976), 119-130.

[29] Lumer, G., and Phillips, R.S., Dissipative operators in a Banach space, Pacific J. Math. 11 (1961), 679-698.

[30] Phillips, R.S., Dissipative operators and hyperbolic systems of partial differential equations, Trans. Amer. Math. Soc. 90 (1959), 193-254.

[31] Powers, R.T., and Sakai, S., Unbounded derivations in operator algebras, J. Funct. Anal. 19 (1975), 81-95.

[32] Pulvinenti, M., and Tirozzi, B., Time evolution of a quantum lattice system, Comm. Math. Phys. 30 (1973), 83-98.

[33] Ruelle, D., Statistical Mechanics: Rigorous Results, W. A. Benjamin, New York-Amsterdam, 1969.

[34] Ruelle, D., Topics in quantum statistical mechanics and operator algebras, preprint, 2001.

[35] Sakai, S., $C^{*}$-Algebras and $W^{*}$-Algebras, Ergebnisse der Mathematik und ihrer Grenzgebiete, Band 60, Springer-Verlag, Berlin-Heidelberg-New York, 1971.

[36] Sakai, S., Recent developments in the theory of unbounded derivations in $C^{*}$-algebras, $C^{*}$ Algebras and Applications to Physics (Los Angeles, 1977) (H. Araki and R.V. Kadison, eds.), Lecture Notes in Mathematics, vol. 650, Springer-Verlag, Berlin-Heidelberg-New York, 1978, pp. 85-122.

[37] Salmhofer, M., and Wieczerkowski, C., Positivity and convergence in fermionic quantum field theory, J. Statist. Phys. 99, (2000), 557-586.

[38] Tomiyama, J., On the projection of norm one in $W^{*}$-algebras, Proc. Japan Acad. 33 (1957), 608-612. 